\title{
A LAGRANGIAN PANEL METHOD IN THE TIME DOMAIN FOR MOVING FREE-SURFACE POTENTIAL FLOWS
}

\author{
J. D'ELÍA ${ }^{1}$, M.A. STORTI ${ }^{1}$, E. OÑATE ${ }^{2}$ AND S.R. IDELSOHN ${ }^{1}$ \\ 1: International Centre for Computational Methods in Engineering (CIMEC) \\ INTEC, UNL-CONICET, Güemes 3450, 3000-Santa Fe, Argentina \\ cimec@arcride.edu.ar, http://venus.arcride.edu.ar/CIMEC \\ 2: Centro Internacional de Métodos Numéricos en Ingeniería (CIMNE) \\ Universidad Politécnica de Catalunya (UPC), Módulo C1 \\ Campus Nord UPC, Gran Capitán s/n, 08034-Barcelona \\ http://www.cimne.upc.es
}

\section{ABSTRACT}

A Lagrangian-type panel method, in the time domain, is proposed for potential flows with a moving free surface. After a spatial semi-discretization, with a low-order scheme, the instantaneous velocity-potential and normal displacement on the moving free surface, are obtained by means of a time-marching scheme. The kinematic and dynamic boundary conditions, at the free surface, are non-lineal restrictions over the related Ordinary Differential Equation system and, for handle them, an alternative Steklov-Poincaré operator technique is proposed. The method is applied to sloshing like flow problems.

KEY WORDS: computational fluid dynamics, free surface flows, Lagrangian panel method, sloshing flow, Steklov-Poincaré operator

\section{INTRODUCTION}

Applications of inviscid free surface flows include a wide variety of phenomena such as, for example, wave-resistance, seakeeping, wave-washing, drift and tidal currents, storm surge, wave propagation and sloshing. The engineering projects require several types of predictive flow models, where the acceptance of a predictive simulation is related to its price to performance ratio, i.e. the computational effort for a simulation run in comparison with the physical relevance of its results and, in general, this price increases with the level of its sophistication. It is well 

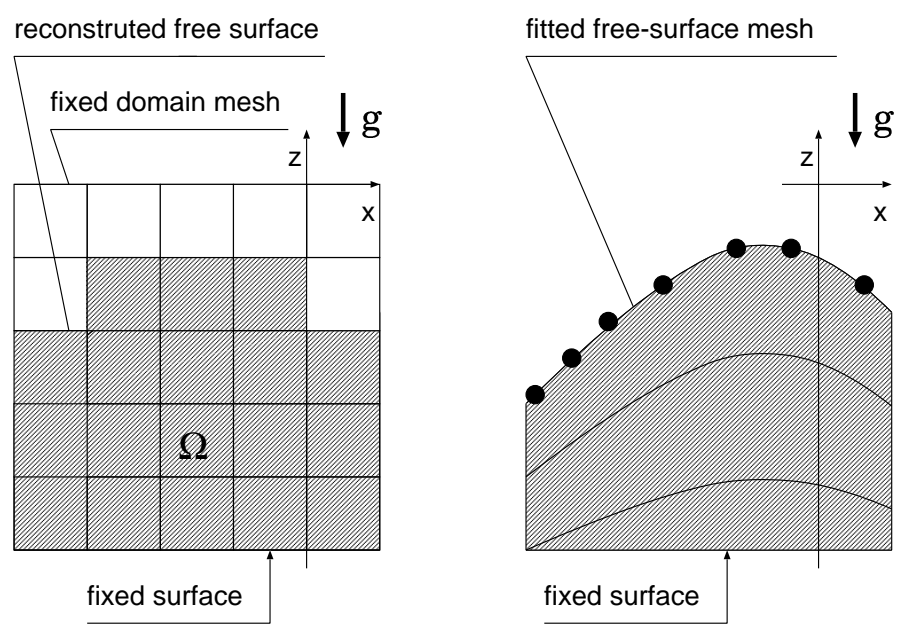

Figure 1: A 2D-flow domain with a free surface discretized by domain-like schemes: Euler-type (left) and Lagrangian-type (right) methods.

known the great variety of scales involved in fluid dynamic phenomena playing a basic role in the applications of scientific and industrial interest.

The panel (boundary element) method is a member of the CFD family which can be used in preliminary design stages, e.g. see Faltinsen (1998). It is based in the discretization of boundary integral equations, which are closely related with the Green function theory, e.g. see Brebbia (1984). As it is well known, this method assumes a potential flow model and it is a practical tool for predicting the pressure field over rather complicated geometries, e.g. see Mueller and Kinnas (1999), or when a parametric study involves an extensive set of numerical test cases, as the transfer functions plots (Response Amplitude Operators RAO), e.g. see Lloyd (1989). Then, it is also possible to shift to another fluid dynamics description level, e.g. Euler and Navier-Stokes solvers, or use mixed strategies, as the viscous-inviscid interaction techniques, e.g. see Williams (1985).

The case of transient free surface flow problems remains as challenging, as is remarked by Khayat et al. (2001). Typically, a boundary-value problem of moving type involves geometrical non-linearities. In contrast to CFD conventional problems, the computational flow domain is partially bounded by a free surface, which is not known a priori, since its shape itself must be computed as part of the solution. In steady-flow the free surface is obtained by an iterative process, but the problem is more difficult when the free surface evolves with time, generating large distortions in the computational flow domain.

Several numerical techniques have been developed for the solution of free surface flows as initial value problems. These techniques are roughly classified by Shy et al. (1996) as Eulerian, Lagrangian and mixed Eulerian-Lagrangian. In Eulerian-like (volume-tracking) approaches, see figure 1, the mesh remains stationary or moves in predetermined manner, the free surface is not explicitly tracked, so it is reconstructed from other field properties, such as the fluid fractions, 


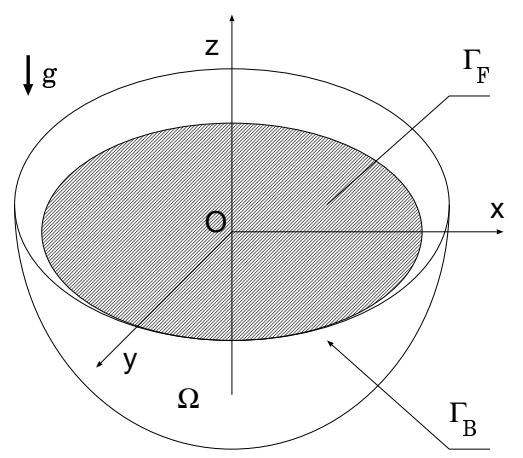

Figure 2: Schematic diagram of a sloshing-like problem: flow domain $\Omega$, rigid surface $\Gamma$ and free surface $\Gamma_{F}$.

and the fluid moves in/out of the computational flow domain. It can handle large displacements without loss of accuracy, but is rather difficult to impose the free boundary conditions, since a lack of a sharp definition, e.g. see Nickell et al. (1974), Siliman and Scriven (1980), Ruschak (1980) and Kawahara and Miwa (1984). In Lagrangian-like (surface-tracking) approaches, the mesh is configured to conform the shape of the free surface and, thus, it adapts continually to it. The free surface is a discontinuity and explicitly we track its evolution, as an $(n-1)$ dimensional entity in an $n$-dimensional space. No modeling is necessary to define the free surface or its effects on the flow field. The grid points move with the local fluid particles, so the free surface is sharply defined but, however, mesh refinement or remeshing usually is necessary for large deformations, e.g. see Bach and Hassager (1985), Ramaswamy and Kawahara (1987). In mixed Eulerian Lagrangian-like approaches, the advantages of both methods are taken into account, e.g. see Chiapada et al. (1996). Also, another mixed approaches are proposed. For example, the "Emplicit" method uses an explicit-implicit time integration oriented to seakeeping ship motions, e.g. see Huang and Sclavounos (1998), while the "Material Point" method uses unconnected Lagrangian points and a background Eulerian mesh for solving fluid-membrane interaction, e.g. see York et al. (2000).

Sometimes, Lagrangian-like approaches can become difficult to implement for three dimensional (3D) flow problems, when domain-based discretizations (as finite elements) are used, while with boundary-based ones (as boundary elements) could be more easy to formulate, together with a more easy adaptive surface remeshing. For potential flows, a typical panel strategy relates the velocity-potential and its normal derivative on points over the boundary surface, so it could be an ideal method for moving-boundary flow problems, where the position and velocity of the free surface is a prime interest quantity.

On the other hand, the "sloshing" flow problems, e.g. see Morand and Ohayon (1995), describe the motion of a liquid enclosed in a rigid and partly filled vessel. There is an interface between the liquid and the gas that fills up the rest of the vessel, see figure 2. This interface 


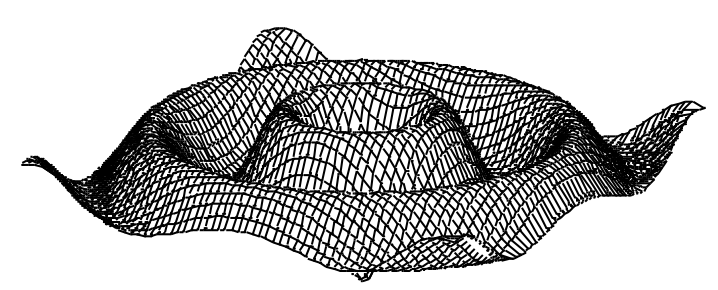

Figure 3: A sloshing oscillation mode, on the free surface of liquid enclosed in a rigid and partly filled vessel.

is a free surface and represents a moving boundary of the enclosed liquid, and has a strong influence on the liquid motion. As it is known, a body enclosing a cavity completely filled with an inviscid, irrotational and incompressible flow, is dinamically equivalent to some other solid body, and whose solution can be found using the Stokes-Jukowski potentials. But, when the liquid has a free surface, there is not such equivalent solid body, since the free surface introduces new degrees of freedom. Nevertheless, for a stable equilibrium position is sufficient that exist other equivalent solid bodies whose equilibrium positions should be also stable. As all enclosed moving system, the liquid motion has discrete natural frequencies and oscillation modes, see figure 3, depending upon of the shape and size of the vessel and amount of the enclosed liquid. When the vessel is subjected to a forced oscillation at a frequency close to a natural "slosh" frequency resonance, we have large displacements of the free surface, for example, holds of crudeoil carrying tankers, large water reservoirs excited by seismic activity or fuel tanks of rocket engines with liquid-propeller. A review of the classical theory is given by Moiseev (1964). For a survey of the related eigenvalue problem, application of the Ritz method and semi-numerical methods, e.g. see Moiseev and Petrov (1966), where eigenfunction expansions for some simple geometries (as parallepiped, circular and annular cylinders) are also given.

Wall pressure estimation for a structural design, has been often based in the Housner (1957) method, where the hydrodynamic pressure is decomposed as the sum of two parts: an impulsive pressure of the liquid moving together with the rigid tank, and a convected pressure of the liquid under sloshing motion at the fundamental sloshing frequency. For the fuel motion for air-plane dynamics by an equivalent spring-mass system, e.g. see Graham and Rodríguez (1952). In liquid-propeller rocket engines, radially compartmented cylindrical fuel tanks are often used, in order to increase the first natural sloshing frequency and reduces the related sloshing liquid mass, e.g. see Bauer (1963). For experimental tests of free and forced vibrations on partially filled spherical tanks, e.g. see Abramson et al. (1963). For the dynamic of flexible liquid storage tanks by finite elements, e.g. see Balendra et al. (1982), Haroun (1983), and by boundary elements, e.g. see Dutta and Laha (2000), Hwang and Ting (1987), while Koh et al. (1998) show a coupled FEM-BEM code for a 3D rectangular liquid storage with flexible walls. In the Dutta and Laha work, a linearized analysis in the frequency domain for a rigid container of arbitrary 
shape is done, and it is solved with a low-order boundary element method, where the oscillation amplitude is assumed to be small enough to allow linearization of the boundary condition at the free surface.

The strategy in this work is to solve the sloshing flow problem, with an adaptive Lagrangianlike panel method in the time domain. After a spatial semi-discretization, with a low-order scheme, the instantaneous velocity-potential and normal displacement on the moving free surface, are obtained by means of a time-marching scheme. The boundary conditions at the free surface, are non-lineal restrictions over the related Ordinary Differential Equation system so that, for handle them, an alternative Steklov-Poincaré operator technique is developed. The position of the moving free surface is updated at each time-step, but only small distortions are permited, so remeshing is not considered in this work.

\section{MATHEMATICAL FORMULATION}

\subsection{The sloshing flow problem}

We consider a free surface performing small oscillations around its hydrostatic equilibrium position, on the free surface of a potential incompressible flow, as sketched in figure 2. An hydrodynamic standard analysis in space $\mathbf{x}=(x, y, z)$ and time $t$ domains gives: the Laplace equation for the velocity-potential $\phi(\mathbf{x}, t)$ in the instantaneous flow domain $\Omega(t)$, no-slip boundary condition at the rigid boundary $\Gamma_{B}(t)$, and the kinematic and dynamic boundary conditions, on the instantaneous free surface $\Gamma_{F}(t)$, for the wave-height $\eta(\mathbf{x}, t)$ and flux $\phi_{, n}=\sigma(\mathbf{x}, t)$, that is,

$$
\begin{array}{ccc}
\nabla^{2} \phi & =0 & \text { in } \Omega(t) ; \\
\phi_{, n} & =0 & \text { at } \Gamma_{B} \\
-\eta_{, t}+n_{z}^{-1} \sigma & =0 & \text { at } \Gamma_{F}(t) \\
\phi_{, t}+\left.\frac{1}{2} \nabla \phi\right|^{2}+g \eta & =0 & \text { at } \Gamma_{F}(t)
\end{array}
$$

where $\mathbf{n}$ is the unit normal of the free surface, $n_{z}$ is its projection on the $z$-axis, positive upwards, and $g$ is the gravity acceleration.

\subsection{Spatial semi-discretization by a panel method}

For a numerical solution in the time-domain $t$, we choose a low-order panel method. First, a semi-discretization in the spatial variable $\mathbf{x}$ is done for an instantaneous geometry, which has a free surface, some fixed surfaces (e.g. the bottom in a ship-like case or the vessel in a sloshinglike one). In this way, we arrive to a system of Ordinary Differential Equations (ODE) for the 
time $t$. Neglecting the gradient term in Eqn. (1.c), the implicit form

$$
\begin{array}{cc}
\mathbf{H}\left(\boldsymbol{\eta}_{S}\right) \mathbf{u}_{1}+\mathbf{G}\left(\boldsymbol{\eta}_{S}\right) \mathbf{u}_{2} & =\mathbf{0} \\
-\boldsymbol{\eta}_{S, t}+\mathbf{n}_{S S}^{-1} \boldsymbol{\sigma}_{S} & =\mathbf{0} \\
\boldsymbol{\phi}_{S, t}+g \boldsymbol{\eta}_{S} & =\mathbf{0}
\end{array}
$$

is obtained, where $\mathbf{n}_{S S}=\operatorname{diag}_{S S}\left(n_{z}\right)$ is a diagonal matrix with the $z$-component of the panel normals, and $\mathbf{u}_{1}, \mathbf{u}_{2}$ are the mixed vectors

$$
\mathbf{u}_{1}=\left[\begin{array}{c}
\phi_{B} \\
\boldsymbol{\sigma}_{S}
\end{array}\right] ; \mathbf{u}_{2}=\left[\begin{array}{c}
\boldsymbol{\sigma}_{B} \\
\boldsymbol{\phi}_{S}
\end{array}\right] ;
$$

while the influence matrices $\mathbf{H}, \mathbf{G}$ are obtained by means of a standard panel discretization for the Laplace equation with Neumann and Dirichlet boundary conditions, the Morino mixed formulation, e.g. see Maître (1988), Morino (1985), Kuo and Morino (1974), which leads the sub-matrices

$$
\mathbf{H}=\left[\begin{array}{ll}
\mathbf{H}_{B B} & \mathbf{H}_{B S} \\
\mathbf{H}_{S B} & \mathbf{H}_{S S}
\end{array}\right] ; \mathbf{G}=\left[\begin{array}{ll}
\mathbf{G}_{B B} & \mathbf{G}_{B S} \\
\mathbf{G}_{S B} & \mathbf{G}_{S S}
\end{array}\right] ;
$$

where the sub-indices $B, S$ denote free and body surface, respectively, and also refer the involved matrix dimensions, that is, $B$ panels on the body and $S$ panels on the free surface. This system, in this form, has a drawback that it is not really an ODE' system, but a Differential and Algebraic Equation (DAE) one, in the sense that we have $2 S+B$ equations with the unknowns $\boldsymbol{\eta}_{S, t}, \boldsymbol{\phi}_{S, t}$ and $\phi_{B}$, that is, of the type

$$
\mathbf{A}\left[\begin{array}{c}
\mathbf{x}, t \\
\mathbf{y}
\end{array}\right]=\mathbf{f}(\mathbf{x}, \mathbf{y}, t)
$$

where $\mathbf{A}$ has a $N \times N$ size, and the vectors $\mathbf{x}, \mathbf{y}$ have the $N_{x}, N_{y}$ lengths, respectively, with $N=N_{x}+N_{y}$. Then, the vector $\mathbf{y}$ of the last $N_{y}$ equations can be eliminated and replaced on the first $N_{x}$ equations, and an ODE system in $\mathbf{x}_{, t}$ could be obtained, but we have developed another strategy based on a Steklov-Poincaré like operator technique in conjunction with a Crank-Nicholson like scheme.

\subsection{The Steklov-Poincaré operator for the Laplace equation}

The Steklov-Poincaré operator technique frequently is used, for example, in $D t N$ absorbing boundary conditions, e.g. see Givoli and Keller (1990), Huan and Thompson (2000), and domain decomposition techniques, e.g. see Quarteroni (1995). For instance, for the Laplace equation in

a domain $\Omega$, under Dirichlet boundary conditions at its boundary $\Gamma$ :

$$
\begin{array}{ccc}
\Delta \phi & =0 & \text { in } \Omega ; \\
\phi & =0 & \text { at } \Gamma_{0} \\
\phi & =\hat{\phi} & \text { at } \Gamma_{\phi}
\end{array}
$$


where $\Gamma=\Gamma_{0} \cup \Gamma_{\phi}$. In the most simple fashion, a one-valued relation between the Dirichlet data $\hat{\phi}$ (over the boundary $\Gamma$ ) and the solution $\phi$ (in the domain $\Omega$ ) is postulated. A similar assumption is postulated for the flux (normal derivative to the boundary surface) $\phi_{, n}$, so these are written as

$$
\begin{aligned}
\phi & =\mathcal{H} \hat{\phi} \\
\phi_{, n} & =\mathcal{S} \hat{\phi}
\end{aligned}
$$

where $\mathcal{H}, \mathcal{S}$ remark that are non-local operators. For all harmonic functions $\nabla^{2}(\ldots)=0$ in the domain $\Omega$, it can been shown that this Steklov-Poincaré operator is linear, symmetric, and positive definite. In effect,

$$
\begin{gathered}
(\psi, \mathcal{S} \phi)=\int_{\Gamma_{\phi}} \psi \mathcal{S} \phi d \Gamma=\int_{\Gamma_{\phi}} \psi \phi_{, n} d \Gamma \\
=\int_{\Gamma_{\phi}}(\psi \nabla \phi) \cdot \hat{\mathbf{n}} d \Gamma=\int_{\Omega}\left[\nabla \psi \cdot \nabla \phi+\psi \nabla^{2} \phi\right] d \Omega=\int_{\Omega} \nabla \psi \cdot \nabla \phi d \Omega .
\end{gathered}
$$

Repiting again Eqn. (8) but interchanging the functions, and taking into account $\nabla^{2} \psi=0$ in the domain $\Omega$,

$$
(\psi, \mathcal{S} \phi)=\int_{\Omega} \nabla \psi \cdot \nabla \phi d \Omega=(\phi, \mathcal{S} \psi) \quad \text { (symmetric) }
$$

and when, in particular, $\psi=\phi$, we have

$$
(\phi, \mathcal{S} \phi)=\int_{\Omega}\|\nabla \phi\|^{2} d \Omega>0 \quad \text { (positive definite) }
$$

where we have been assumed that $\|\nabla \phi\|$ is squared integrable. Also, it is bijective. In effect, if we suppose that we have $\mathcal{S} \phi_{1}=\mathcal{S} \phi_{2}$ and $\phi^{\prime}=\phi_{1}-\phi_{2}$, then $\mathcal{S} \phi^{\prime}=0$ and $\left(\phi^{\prime}, \mathcal{S} \phi^{\prime}\right)=0$, so that, from Eqn. (10),

$$
0=\left(\phi^{\prime}, \mathcal{S} \phi^{\prime}\right)=\int_{\Omega}\left\|\nabla \phi^{\prime}\right\|^{2} d \Omega
$$

but, in such case, $\phi^{\prime}=$ cnst and then it is sufficient that there are some portion of the boundary with Dirichlet condition for arrive to $\phi^{\prime} \equiv 0$. A standard FEM-like discretization for this operator gives a (full) symmetric and positive defined system matrix $\hat{\mathbf{H}}$, obtaining $\phi=\mathbf{H} \hat{\boldsymbol{\phi}}$ but, on the other hand, a standard BEM-like one gives a near (full) symmetric and positive defined one. In the context of domain decomposition techniques, $\hat{\mathbf{H}}$ is often called the Schur complement, e.g. see Cottle (1974), whose functional counterpart is, precisely, the Steklov-Poincaré operator, e.g. see Quarteroni (1995), Serón and Sabadell (2000).

An engineering application of the Steklov-Poincaré operator, is the time-domain evolution of a potential flow with a moving free surface by a Lagrangian-type panel method. In such case, 
it is immediate to show that such problem always will gives a wave-like equation. In effect, the system (1.c-d) on the free surface, at first order, is

$$
\begin{array}{rlr}
\eta_{, t}-n_{z}^{-1} g \phi_{, n} & =0 & \text { at } \Gamma_{F} ; \\
\phi_{, t}+g \eta & =0 & \text { at } \Gamma_{F} ;
\end{array}
$$

while (1.a-b)

$$
\begin{aligned}
& \nabla^{2} \phi=0 \quad \text { in } \Omega(t) ; \\
& \phi_{, n}=0 \quad \text { at } \Gamma_{B} ;
\end{aligned}
$$

are working as "restrictions" for the dynamics of the moving free surface. Eliminating the wave-height $\eta$, we obtain the (reduced) second order differential equation

$$
\phi_{, t t}-n_{z}^{-1} g \phi_{, n}=0 \quad \text { at } \Gamma_{F}
$$

introducing the Steklov-Poincaré operator for the normal derivative $\phi_{, n}=S \phi$, we have

$$
\phi, t t+\mathcal{M}^{2} \phi=0 ; \quad \text { at } \Gamma_{F} ;
$$

where $\mathcal{M}^{2}=n_{z}^{-1} g \mathcal{S}$, which have wave-like solutions $\phi=A e^{i \mathcal{M} t}$. For a linearized boundary problem, a standard Fourier analysis can be done and gives an Helmholtz-like equation, whose numerical solution has a rather expensive computational cost. For this reason, we discard second-order approaches and work with a first-order system, for a time-marching scheme of the moving free-surface.

\subsection{Numerical implementation}

A standard discretization of Eqn. (2) by finite differences between the times $n, n+1$, present and next, respectively, we have

$$
\left[\begin{array}{c}
\mathbf{F}_{1}^{n, n+1} \\
\mathbf{F}_{2}^{n, n+1} \\
\mathbf{F}_{3}^{n, n+1} \\
\mathbf{F}_{4}^{n, n+1}
\end{array}\right]=\left[\begin{array}{c}
\alpha \mathbf{R}_{B}^{n+1}+\beta \mathbf{R}_{B}^{n} \\
\alpha \mathbf{R}_{S}^{n+1}+\beta \mathbf{R}_{S}^{n} \\
-h^{-1} \mathbf{n}_{S S}\left(\boldsymbol{\eta}_{S}^{n+1}-\boldsymbol{\eta}_{S}^{n}\right)+\alpha \boldsymbol{\sigma}_{S}^{n}+\beta \boldsymbol{\sigma}_{S}^{n+1} \\
h^{-1} g^{-1}\left(\boldsymbol{\phi}_{S}^{n+1}-\boldsymbol{\phi}_{S}^{n}\right)+\alpha \boldsymbol{\sigma}_{2}^{n}+\beta \boldsymbol{\eta}_{S}^{n+1}
\end{array}\right]=\left[\begin{array}{c}
\mathbf{0} \\
\mathbf{0} \\
\mathbf{0} \\
\mathbf{0}
\end{array}\right]
$$

where $h$ is the temporal step, $\alpha$ and $\beta=1-\alpha$ are the weights of the integration scheme, e.g. $\alpha=1$ for backward-Euler and $\alpha=0$ for Crank-Nicholson. The residues, on the body $\mathbf{R}_{B}$ and on the free surface $\mathbf{R}_{S}$, are given by

$$
\begin{gathered}
\mathbf{R}_{B}=\mathbf{H}_{B B} \boldsymbol{\phi}_{B}+\mathbf{H}_{B S} \boldsymbol{\sigma}_{S}+\mathbf{G}_{B B} \boldsymbol{\sigma}_{B}+\mathbf{G}_{B S} \boldsymbol{\phi}_{S} ; \\
\mathbf{R}_{S}=\mathbf{H}_{S B} \boldsymbol{\phi}_{B}+\mathbf{H}_{S S} \boldsymbol{\sigma}_{S}+\mathbf{G}_{S B} \boldsymbol{\sigma}_{B}+\mathbf{G}_{S S} \boldsymbol{\phi}_{S} ;
\end{gathered}
$$

We see that the Eqn. (16) represents a non-lineal equation like

$$
\mathbf{F}^{n, n+1} \equiv \mathbf{F}\left(\mathbf{U}^{n}, \mathbf{U}^{n+1}\right)=\mathbf{0}
$$


with the state vector $\mathbf{U}=\left(\boldsymbol{\phi}_{B}, \boldsymbol{\phi}_{S}, \boldsymbol{\eta}_{S}, \boldsymbol{\sigma}_{S}\right)$. In the spirit of the semi-implicit schemes, we perform a Taylor expansion of Eqn. (18), near the point $\left(\mathbf{U}^{n}, \mathbf{U}^{n+1}\right)$, that is,

$$
\mathbf{F}\left(\mathbf{U}^{n+1}, \mathbf{U}^{n}\right)=\mathbf{F}\left(\mathbf{U}^{n}, \mathbf{U}^{n}\right)+\mathbf{J}^{n} \Delta \mathbf{U}^{n}=\mathbf{0}
$$

where

$$
\mathbf{J}^{n}=\left.\frac{\partial \mathbf{F}}{\partial \mathbf{U}^{n+1}}\right|_{\mathbf{U}^{n}, \mathbf{U}^{n}} ;
$$

is the Jacobian and $\Delta \mathbf{U}^{n}=\mathbf{U}^{n+1}-\mathbf{U}^{n}$. Then

$$
\mathbf{J}^{n} \Delta \mathbf{U}^{n}=-\mathbf{F}\left(\mathbf{U}^{n}, \mathbf{U}^{n}\right)
$$

is a system whose solution will give the increments for the wave-height and the (perturbation) velocity-potential. A straightforward computation gives the lineal system (omitting the supraindex $n)$ :

$$
\left[\begin{array}{cccc}
\mathbf{H}_{B B} & \mathbf{G}_{B S} & \mathbf{X}_{B S} & \mathbf{H}_{B B} \\
\mathbf{H}_{S B} & \mathbf{G}_{S S} & \mathbf{X}_{S S} & \mathbf{H}_{S B} \\
\mathbf{0} & \mathbf{0} & -h^{-1} \mathbf{n}_{S S} & \alpha \mathbf{I}_{S S} \\
\mathbf{0} & h^{-1} g^{-1} \mathbf{I}_{S S} & \alpha \mathbf{I}_{S S} & \mathbf{0}
\end{array}\right]\left[\begin{array}{c}
\Delta \boldsymbol{\phi}_{B} \\
\Delta \boldsymbol{\phi}_{S} \\
\Delta \boldsymbol{\eta}_{S} \\
\Delta \boldsymbol{\sigma}_{S}
\end{array}\right]=\left[\begin{array}{c}
-\mathbf{F}_{1} \\
-\mathbf{F}_{2} \\
-\mathbf{F}_{3} \\
-\mathbf{F}_{4}
\end{array}\right] ;
$$

where

$$
\begin{aligned}
& \mathbf{X}_{B B}=\partial \mathbf{F}_{1} / \partial \boldsymbol{\eta}_{S} ; \\
& \mathbf{X}_{B S}=\partial \mathbf{F}_{2} / \partial \boldsymbol{\eta}_{S} ;
\end{aligned}
$$

$\mathbf{I}_{S S}$ is the identity matrix, and $\mathbf{F}=\left[\begin{array}{llll}\mathbf{F}_{1} & \mathbf{F}_{2} & \mathbf{F}_{3} & \mathbf{F}_{4}\end{array}\right]^{T}$ is a source vector with the components

$$
\begin{aligned}
& \mathbf{F}_{1}=\varepsilon \mathbf{R}_{B} ; \\
& \mathbf{F}_{2}=\varepsilon \mathbf{R}_{S} ; \\
& \mathbf{F}_{3}=+\gamma \boldsymbol{\sigma}_{S} ; \\
& \mathbf{F}_{4}=-\gamma \boldsymbol{\eta}_{S} ;
\end{aligned}
$$

and its the first term in the Taylor expansion (19), $\gamma=\alpha+\beta$, with $\varepsilon=\gamma / \alpha$ if $\alpha \neq 0$, and $\varepsilon=1$ otherwise. The $\mathbf{X}_{B B}$ and $\mathbf{X}_{B S}$ matrices are related with the derivatives of the influence matrices and, in this work, these are neglected, so only small distortions on the moving free surface are allowed. The Eqn. $(22)$ has $2(B+S)$ equations and, from a computational cost view-point, we do a partial reduction in the number of unknowns. From the sub-system (22, c-d) we obtain

$$
\begin{aligned}
& \Delta \boldsymbol{\eta}_{S}=-h \mathbf{n}_{S S}^{-1}\left(-\mathbf{F}_{3}-\alpha \Delta \boldsymbol{\sigma}_{S}\right) \\
& \Delta \boldsymbol{\phi}_{S}=\quad+h g\left(-\mathbf{F}_{4}-\alpha \Delta \boldsymbol{\eta}_{S}\right)
\end{aligned}
$$

opening parenthesis in Eqn. (25), we have

$$
\begin{array}{ccc}
\Delta \boldsymbol{\eta}_{S} & = & +h \mathbf{n}_{S S}^{-1} \mathbf{F}_{3}+\alpha h \mathbf{n}_{S S}^{-1} \Delta \boldsymbol{\sigma}_{S} ; \\
\Delta \boldsymbol{\phi}_{S} & = & -\alpha h^{2} g \mathbf{n}_{S S}^{-1} \mathbf{F}_{3}-h g \mathbf{F}_{4}-\alpha^{2} h^{2} g \mathbf{n}_{S S}^{-1} \Delta \boldsymbol{\sigma}_{S} ;
\end{array}
$$


and replacing in Eqn. (22, a-b), results the reduced system

$$
\left[\begin{array}{cc}
\mathbf{H}_{B B} & \tilde{\mathbf{H}}_{B S} \\
\mathbf{H}_{S B} & \tilde{\mathbf{H}}_{S S}
\end{array}\right]\left[\begin{array}{c}
\Delta \boldsymbol{\phi}_{B} \\
\Delta \boldsymbol{\sigma}_{S}
\end{array}\right]=\left[\begin{array}{c}
-\mathbf{B}_{1} \\
-\tilde{\mathbf{B}}_{2}
\end{array}\right]
$$

with the source vectors

$$
\begin{aligned}
& \mathbf{B}_{1}=-\mathbf{F}_{1}-\mathbf{G}_{B S}\left(\alpha \mathbf{M}_{S S} \mathbf{F}_{3}+\mathbf{L}_{S S} \mathbf{F}_{4}\right)+\mathbf{X}_{B S} \mathbf{N}_{S S} \mathbf{F}_{3} \\
& \mathbf{B}_{2}=-\mathbf{F}_{2}-\mathbf{G}_{S S}\left(\alpha \mathbf{M}_{S S} \mathbf{F}_{3}+\mathbf{L}_{S S} \mathbf{F}_{4}\right)-\mathbf{X}_{S S} \mathbf{N}_{S S} \mathbf{F}_{3}
\end{aligned}
$$

and the modified sub-matrices

$$
\begin{aligned}
\tilde{\mathbf{H}}_{B S} & =\mathbf{H}_{B S}-\alpha^{2} \mathbf{G}_{B S} \mathbf{M}_{S S}+\alpha \mathbf{X}_{B S} \mathbf{N}_{S S} ; \\
\tilde{\mathbf{H}}_{S S} & =\mathbf{H}_{S S}-\alpha^{2} \mathbf{G}_{S S} \mathbf{M}_{S S}+\alpha \mathbf{X}_{S S} \mathbf{N}_{S S} ;
\end{aligned}
$$

where

$$
\begin{aligned}
& \mathbf{M}_{S S}=h^{2} g \mathbf{n}_{S S}^{-1} ; \\
& \mathbf{N}_{S S}=h \mathbf{n}_{S S}^{-1} ; \\
& \mathbf{L}_{S S}=h g \mathbf{I}_{S S} .
\end{aligned}
$$

Introducing the auxiliary vectors

$$
\mathbf{u}_{3}=\left[\begin{array}{c}
\mathbf{0}_{B} \\
\alpha \mathbf{M}_{S S} \mathbf{F}_{\mathbf{3}}
\end{array}\right] ; \mathbf{u}_{4}=\left[\begin{array}{c}
\mathbf{0}_{B} \\
\mathbf{L}_{S S} \mathbf{F}_{4}
\end{array}\right] ;
$$

the block diagonal matrices,

$$
\mathbf{U}_{5}=\operatorname{diag}\left[\begin{array}{ll}
\mathbf{0}_{B B} & \alpha^{2} \mathbf{M}_{S S}
\end{array}\right] ; \mathbf{U}_{6}=\operatorname{diag}\left[\begin{array}{ll}
\mathbf{0}_{B B} & \alpha \mathbf{N}_{S S}
\end{array}\right]
$$

and the intermediary vectors

$$
\mathbf{s}=\left[\begin{array}{c}
\Delta \boldsymbol{\phi}_{B} \\
\Delta \boldsymbol{\sigma}_{S}
\end{array}\right] ; \mathbf{v}_{1}=\left[\begin{array}{c}
\mathbf{0}_{B} \\
\boldsymbol{\sigma}_{S}
\end{array}\right] ; \mathbf{v}_{2}=\left[\begin{array}{c}
\mathbf{0}_{B} \\
\boldsymbol{\phi}_{S}
\end{array}\right] ; \mathbf{v}_{3}=\left[\begin{array}{c}
\mathbf{0}_{B} \\
\mathbf{N}_{S S} \mathbf{F}_{3}
\end{array}\right] ;
$$

we can re-write the reduced system (27-29) in a more compact fashion as

$$
\left(\mathbf{H}-\mathbf{G U}_{5}+\mathbf{X \mathbf { U } _ { 6 }}\right) \mathbf{s}=-\mathbf{H} \mathbf{u}_{1}-\mathbf{G}\left(\mathbf{u}_{2}-\mathbf{u}_{3}-\mathbf{u}_{4}\right)-\mathbf{X} \mathbf{v}_{3} .
$$

So, first we computate Eqns. (24, 31-33), then we solve the system (34) and, finally, advance to next time, that is,

$$
\begin{aligned}
& \boldsymbol{\phi}_{B}^{n+1}=\boldsymbol{\phi}_{B}^{n}+\Delta \boldsymbol{\phi}_{B}^{n} \quad ; \\
& \boldsymbol{\phi}_{S}^{n+1}=\boldsymbol{\phi}_{S}^{n}+\Delta \boldsymbol{\phi}_{S}^{n} \quad ; \\
& \boldsymbol{\eta}_{S}^{n+1}=\boldsymbol{\eta}_{S}^{n}+\Delta \boldsymbol{\eta}_{S}^{n}
\end{aligned}
$$



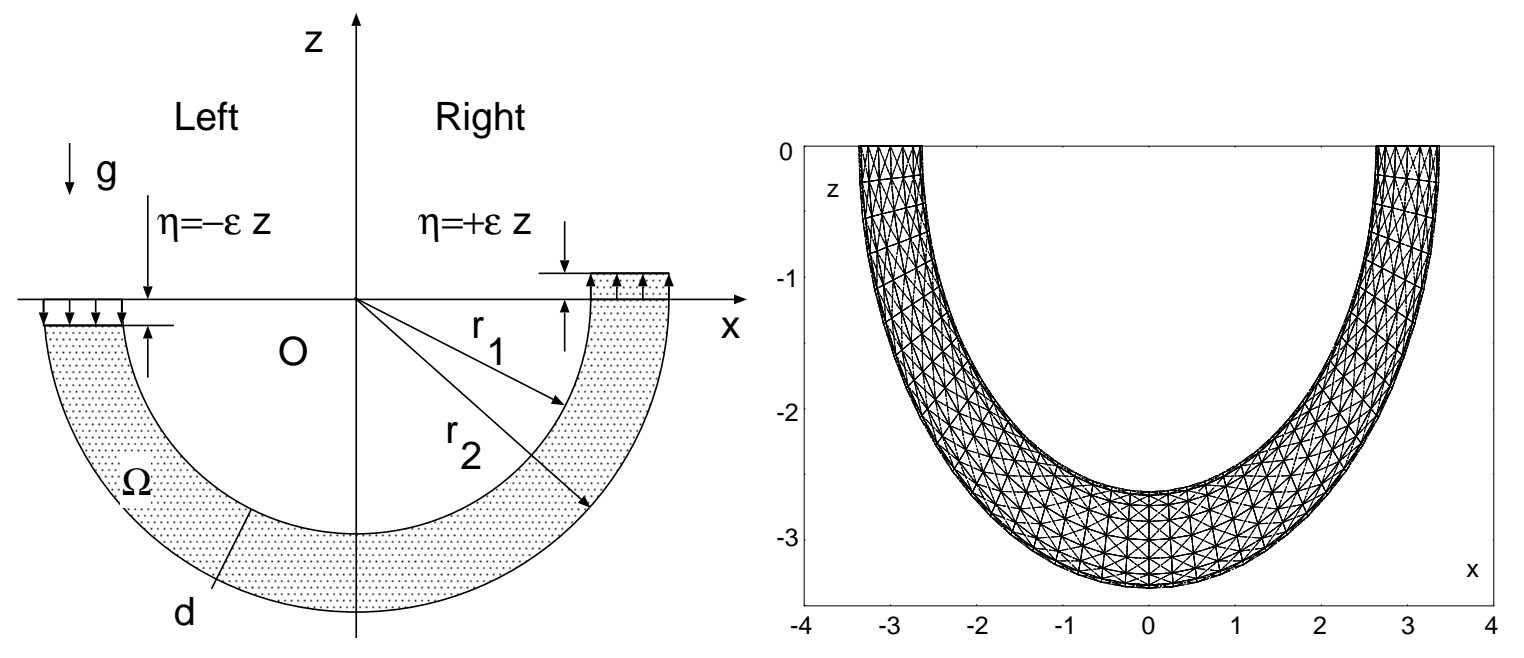

Figure 4: Left: a vertical U-tube of mean radius $r_{m}=\left(r_{1}+r_{2}\right) / 2$ and aspect relation $\mu=r_{m} / d$, and partially filled with a liquid, with a small sloshing motion. Right: vertical $x z$-view of a 3D panel mesh, with $r_{m} \approx 3$ and $\mu \approx 4$.

\section{NUMERICAL EXAMPLES}

The position of the moving free surfaces in the following numerical examples are updated at each time-step but only small distortions are permited, so remeshing is not necessary. The infinity depth case will be assumed for the dispersion relation in all cases, that is, $K=\omega^{2} / g$, where $K$ is the wave-number, $\omega$ is the angular frequency and $g$ is the gravity acceleration.

\subsection{Vertical sloshing in a U-tube}

A rigid U-tube partially filled with a liquid is considered. On its free surface, a small perturbation in its hydrostatic equilibrium height is introduced, in such way that vertical oscillations will develop. As the dissipation effects are neglected, this natural mode state remains in time without attenuation. For a geometrical description, we choose a vertical semi-toroidal domain, with internal and external radius $r_{1}, r_{2}$, respectively, where the (circular) flow section $S$ has a diameter $d=r_{2}-r_{1}$, see figure 4 (left). The mean radius and aspect relation are $r_{m}=\left(r_{1}+r_{2}\right) / 2$ and $\mu=r_{m} / d$, respectively. At the initial time $t=0$ we impose a vertical displacement on each free surface, small enough, and in opposite phase, that is, $\boldsymbol{\eta}_{L}=+\varepsilon \mathbf{z}$ at the left free surface, and $\boldsymbol{\eta}_{R}=-\varepsilon \mathbf{z}$ at the right one, where $0<\varepsilon<1$, and $z$ is the vertical, see figure 4 , left. The natural sloshing wave-number and period can be estimated as $K_{s}=2 / L_{s}$ and $T_{s}=2 \pi\left(g K_{s}\right)^{-1 / 2}$, where $L_{s}=\pi r_{s}$ is a mean perimeter from some radius $r_{s}$, between $r_{1}$ and $r_{2}$.

We choose a mean radius $r_{m} \approx 3$, an aspect relation $\mu \approx 4$ and a gravity acceleration $g=1 / 2$. We have considered 3 meshes of 240,832 and 1408 panels, see figure 4 , right, for a 

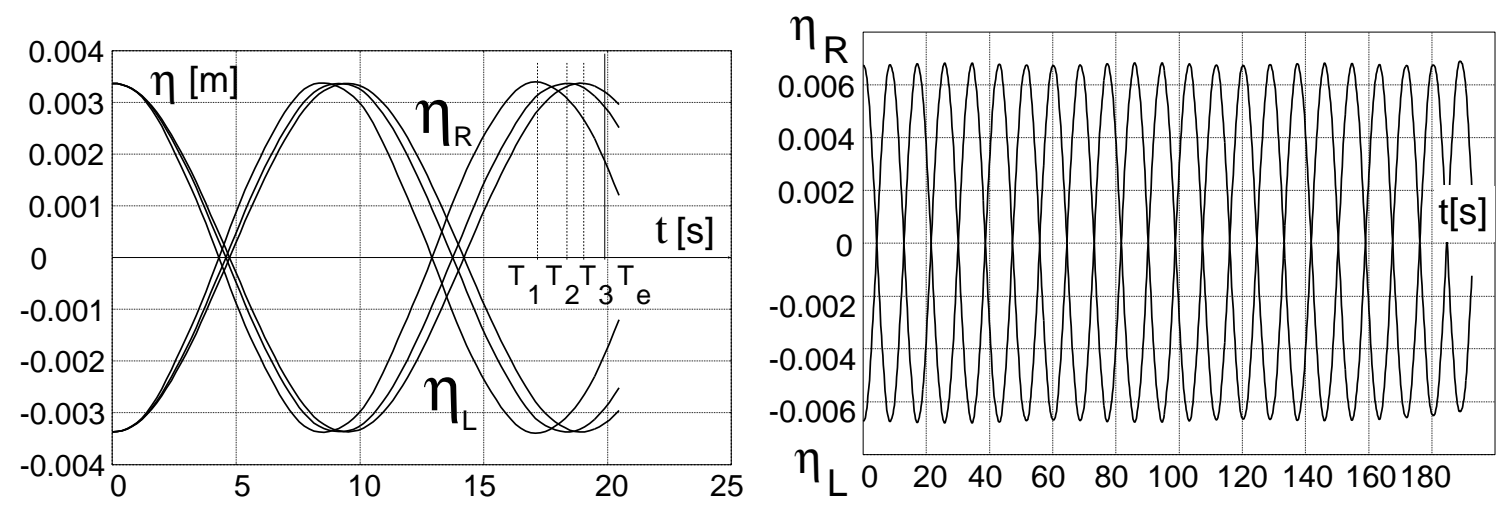

Figure 5: Computed sloshing natural periods for an U-tube from the plots of the wave-height $\eta_{L}(t), \eta_{R}(t)$, at left and right free surface of the U-tube, respectively, as a function of time $t$ : the first period (at the left); the first 15 periods (at the right). Numerical dissipative effects are not perceived.

\begin{tabular}{|c|c|}
\hline computation with & sloshing natural period $T$ [s.] \\
\hline the internal radius $r_{1}$ & 18.07 \\
240 panels & 17.09 \\
832 panels & 18.48 \\
1408 panels & 18.87 \\
the mean radius $r_{m}$ & 19.87 \\
the external radius $r_{2}$ & 20.47 \\
\hline
\end{tabular}

Table 1: Natural sloshing periods on an U-tube: minimum $T_{1}$, mean $T_{m}$ and maximum $T_{2}$ ones (computed with the internal $r_{1}$, mean $r_{m}$ and external $r_{2}$ radii, respectively), compared with those obtained from a panel computation.

vertical $x z$-view. In figure 5 (left), we shown a period of the wave-height $\eta(t)$ plot in the middle sector of each free surface and obtained with the 3 meshes. In figure 5, right, 15 periods are plotted, where numerical dissipative effects are not perceived. The natural sloshing periods: minimum $T_{1}$, mean $T_{m}$ and maximum $T_{2}$ ones (computed with the internal $r_{1}$, mean $r_{m}$ and external $r_{2}$ radii, respectively), and those obtained from a panel computation are compared in Table 1 where, except for the first mesh, the panel estimations are between the mimimum and mean periods.

\subsection{Annular sloshing in a circular cylinder}

A circular cylinder of radius $R$ and depth $H$ enough for neglecting the bottom effects, is considered, see figure 6 , left. The cylinder is assumed to be rigid and it is partially filled. When a perturbation is introduced at the center of its free surface, annular surface waves are generated. 

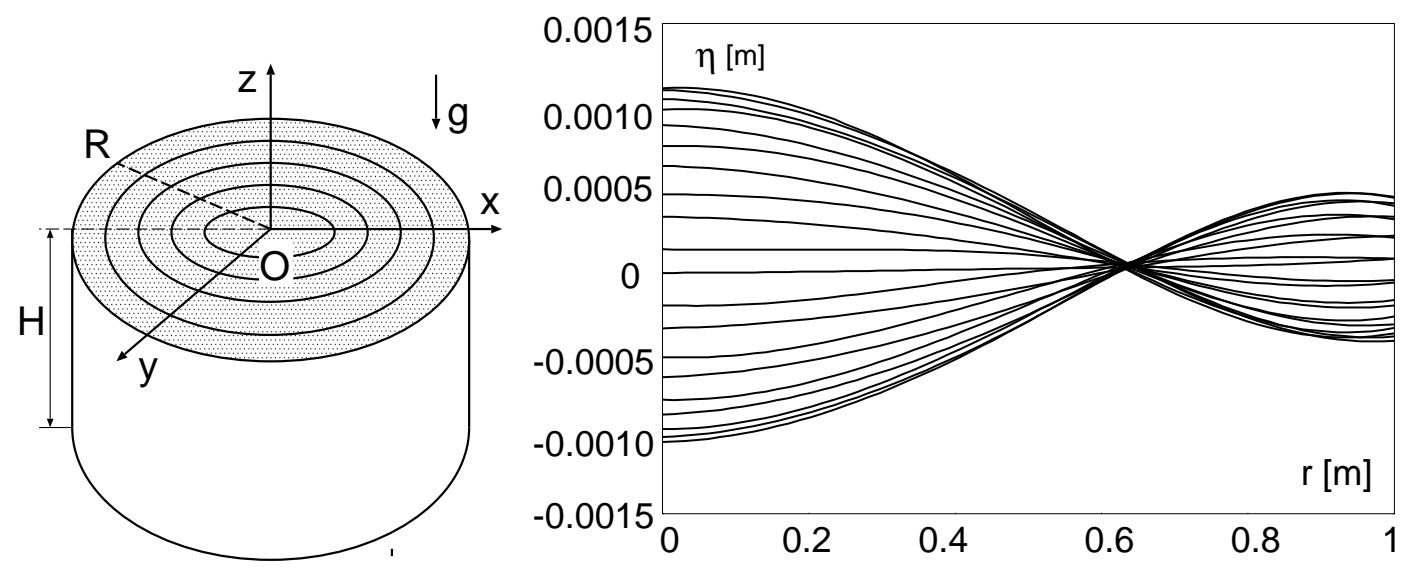

Figure 6: Left: sketch for annular sloshing waves on the free surface of a depth circular cylinder. Right: sloshing mode $\eta(r, t)$, as a function of the radius $r$ and the time $t$, in the first natural sloshing mode, computed by the present method. The analytic ones are proportionals to the Bessel function of first kind and zero-order $J_{0}(K r)$.

Exciting one natural sloshing mode, a standing wave will be obtained. The initial boundary condition imposed at $t=0$ is a natural sloshing mode with radial symmetry. This is a $3 \mathrm{D}$ case which can be solved by an analytic calculation, e.g. see Appendix for a re-derivation, leads the eigen-functions

$$
\Phi_{\alpha}(r, \theta, z, t)=A J_{0}\left(K_{\alpha} r\right) e^{K_{\alpha} z} e^{i \omega t} \quad ; \quad \text { for } z \leq 0
$$

where $J_{0}(K r)$ is the Bessel function of first kind and zero order, $A$ is the amplitude of the oscillation, $x=K r$ is the non-dimensional radial coordinate, $x_{\alpha}$ are the zeros of the Bessel function of first kind and first order $J_{1}(x)$, for instance, $x_{\alpha}=\{3.832,7.016,10.173,13.324, \ldots\}$. The natural sloshing wave-numbers and frequencies are given by $K_{\alpha}=x_{\alpha} / R$ and $\omega_{\alpha}=\sqrt{g x_{\alpha} / R}$, respectively. Simple annular sloshing waves with null radial velocity at $r=R$ and $-H \leq z \leq 0$ are obtained, when the wall is localized at the zeros of $J_{1}(x)$, so there is not necessary to use a superposition principle. A radius $R=1$ and a depth $H=5$ are adopted here.

In figure 7 we show an horizontal $x y$ and vertical $x z$-views of a typical panel mesh. The first sloshing mode $\eta(r, t)$, as a function of the radius $r$ and the time $t$, and obtained with the proposed method, is shown in figure 6 , right, which are well-compared with the analytical ones. In figure 8, 1 and 4 periods of the wave-height $\eta(t)$, in the middle zone of the free surface, are included. The natural sloshing period estimated by the panel code is $T_{B E M} \approx 4.2943$ sec., while the analytic one is $T_{a 1} \approx 4.5394$ sec., with a relative error of $e_{r} \approx-5 \%$.

\subsection{Azimuthal and radial sloshing in an annular cylinder}

A vertical cylinder with an annular base and depth $H$ enough for neglecting the bottom effects, is considered, see figure 9 . The internal and external radii of the annular circular base are $r_{1}$ 

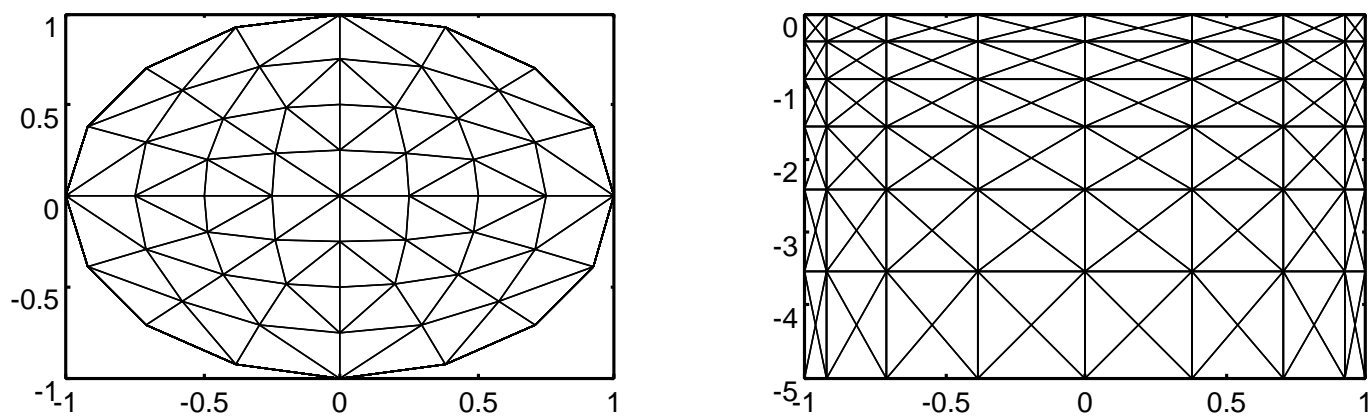

Figure 7: Views of a 3D panel mesh for computing sloshing flow in a circular cylinder.
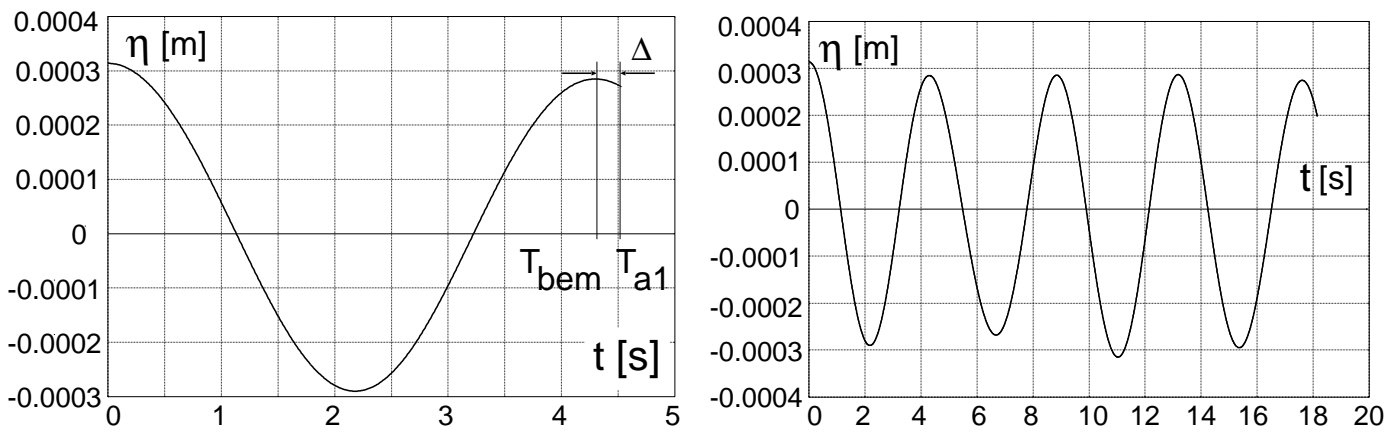

Figure 8: Natural sloshing periods from the plot of the wave-height $\eta(t)$ on a circular cylinder: left: the first period; right: the first 4 ones. Numerical dissipative effects are not perceived.

and $r_{2}$, respectively. The cylinder is assumed to be rigid and it is partially filled. The initial boundary condition imposed at $t=0$, is a natural sloshing mode (azimuthal or radial) so, without physical or numerical dissipation, standing waves will be obtained. A quasi-2d description can be obtained when we consider a thin radial fete, of mean thickness $r_{m} d \theta$, small enough, and $\Delta r / r_{m} \ll 1$, where $r_{m}=\left(r_{1}+r_{2}\right) / 2$ and $\Delta r=r_{2}-r_{1}$ are the mean radius and radial thickness of the annular base, respectively.

The azimuthal-sloshing mode is interesanting due to its simplicity, since it is a stationary wave along the circunferential perimeter, there is not discontinuities in its propagation path, so
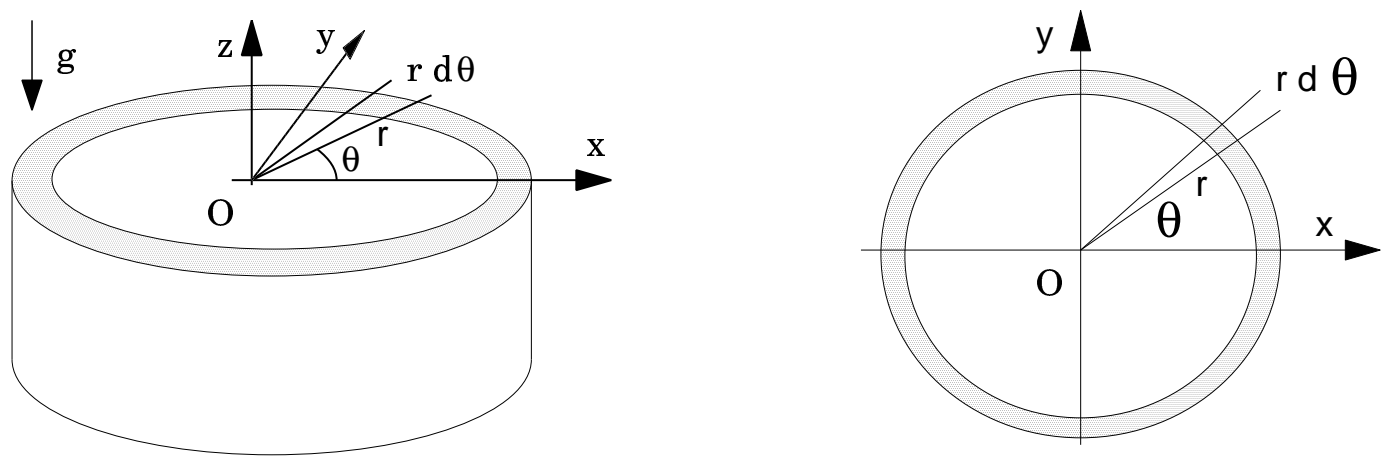

Figure 9: Sketch for a vertical annular cylinder partially filled with a liquid. 

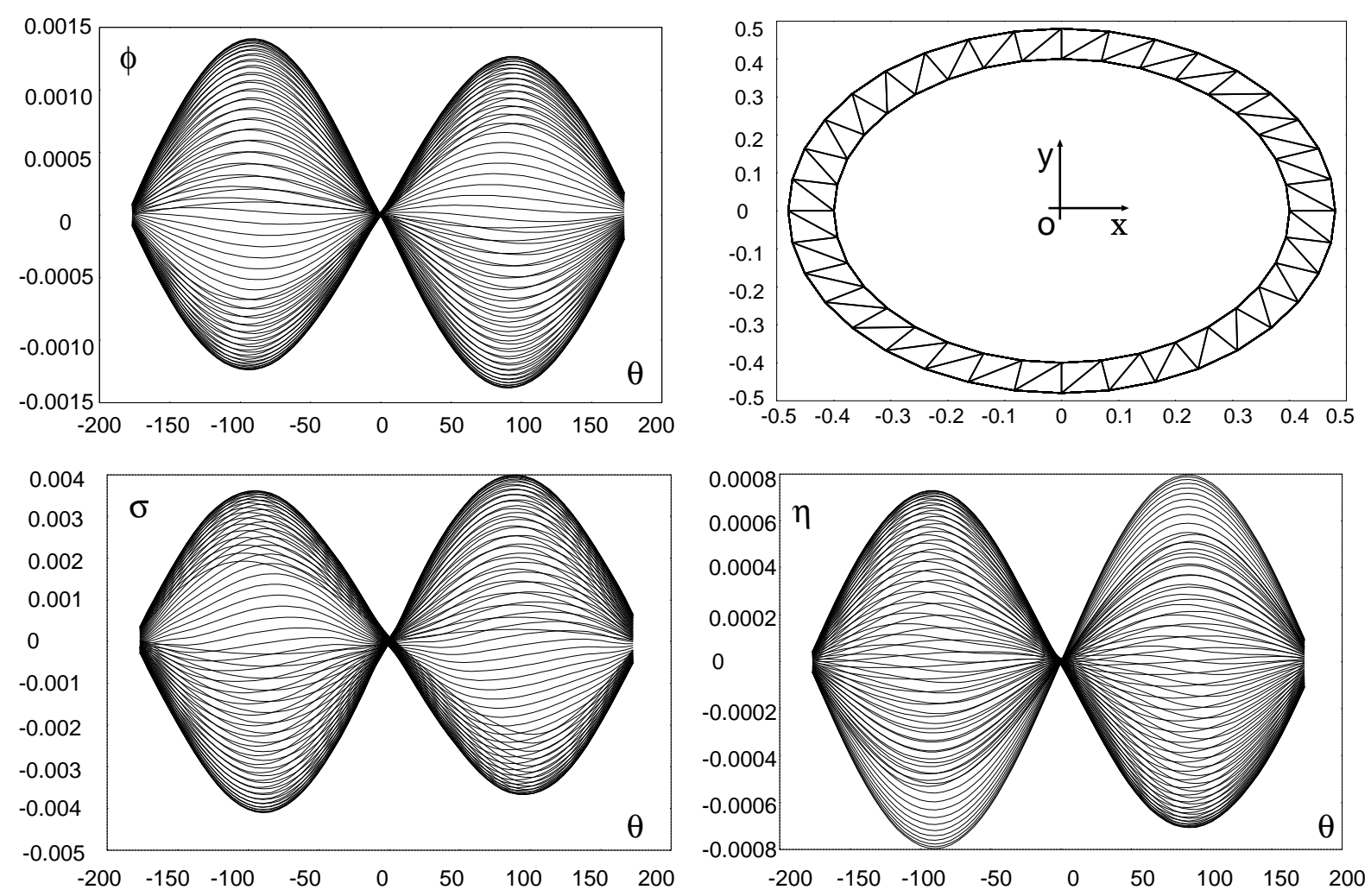

Figure 10: Azimuthal natural sloshing mode in a vertical annular cylinder when, as initial displacement $\eta_{0}$, the natural first azimuthal sloshing mode is imposed: velocity-potential $\phi\left(r_{m}, \theta\right)$, flux $\sigma\left(r_{m}, \theta\right)$ and wave-height $\eta\left(r_{m}, \theta\right)$ at the moving free surface, as a function of the azimuthal angle $\theta$, at the mean radius $r_{m}=\left(r_{1}+r_{2}\right) / 2$.

there is not necessary to implement special tricks for mesh border effects, e.g. "artificial-beaches" or some kind of absorbing boundary conditions. In Fig. 10 we show the time evolution of the first azimuthal-sloshing mode obtained with the proposed method for the velocity-potential, flux and wave-height on the moving free surface.

On the other hand, the radial-sloshing mode is a bit more complicated due the effects caused by the vertical walls at $r_{1}$ and at $r_{2}$. Nevertheless, as the initial displacement is one of the stationary radial sloshing modes, with null radial-velocity at the vertical walls, then, there is not necessary to impose radiation-like boundary conditions. In Fig. 11 we show the time evolution of the first radial-sloshing mode obtained with the proposed method for the velocity-potential, flux and wave-height on the moving free surface.

\section{CONCLUSIONS}

This paper has shown a Steklov-Poincaré technique for solving a Lagrangian-type panel method, for potential flows with a moving free surface. As a special case, the "sloshing" flow problem has been considered. It have been found good agreement with analytic solutions for the natural 

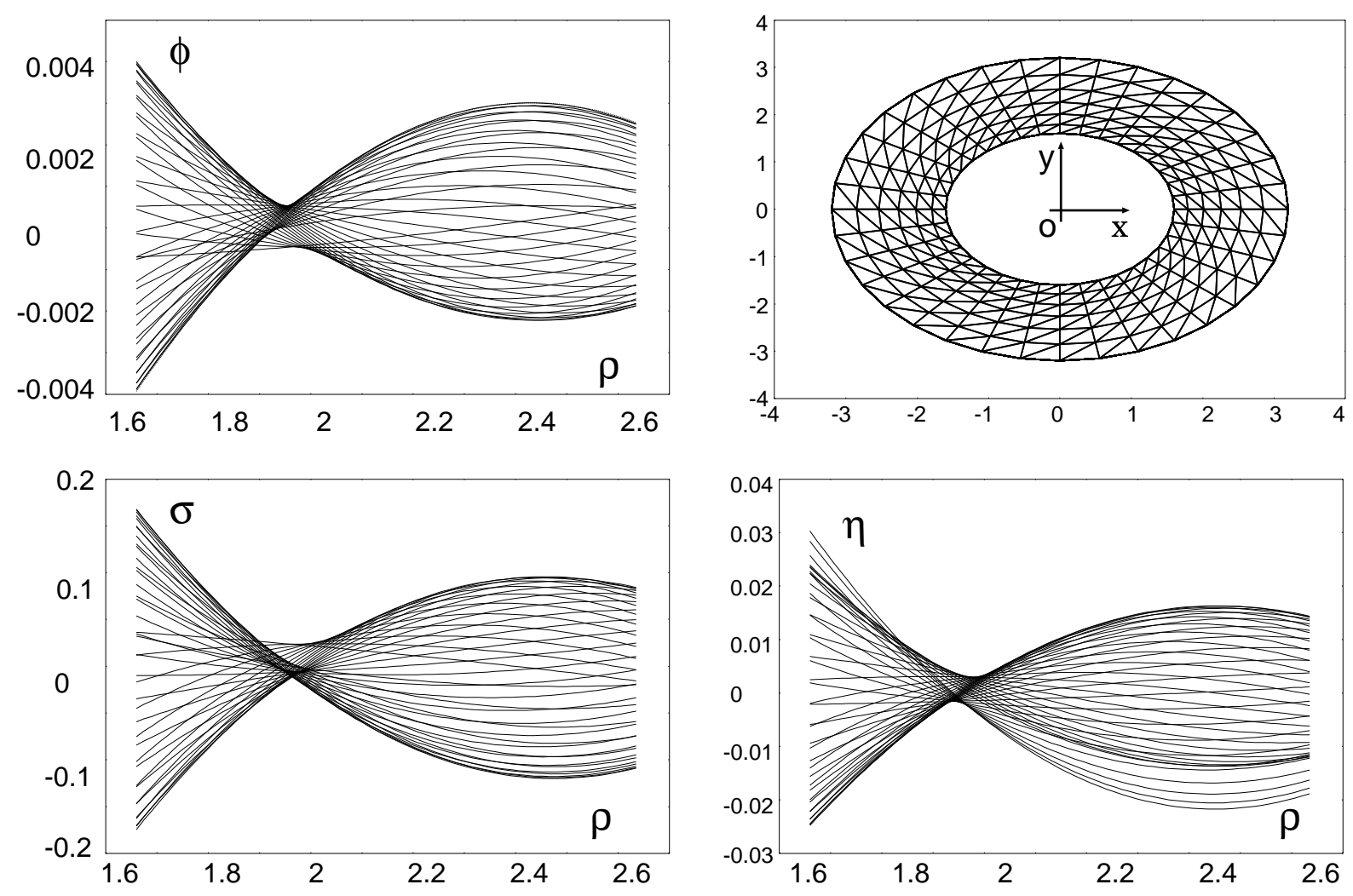

Figure 11: Radial natural sloshing mode in a vertical annular cylinder when, as initial displacement $\eta_{0}$, the natural first radial sloshing mode is imposed: velocity-potential $\phi\left(r, \theta_{k}\right)$, flux $\sigma\left(r, \theta_{k}\right)$ and wave-height $\eta\left(r, \theta_{k}\right)$ at the moving free surface, as a function of the radius $r$, at a fixed azimuthal angle $\theta$.

sloshing frequencies and shape modes. Next stages in the development should include: radiation boundary conditions, for seakeeping-like flow problems, and a remeshing strategy for large distortions on the evolving free surface of non-steady flows.

\section{ACKNOWLEDGEMENTS}

This work was started during the CONICET-postdoctoral stay of the first author at the International Center for Numerical Methods in Engineering (CIMNE, Barcelona) and supported through grants CONICET-PIP-198/98 (Germen-CFD), SECyT-FONCyT-PICT-51 (Germen) and CAI+D-UNL-94-004-024. The work was partially performed with the Free Software Foundation /GNU-Project resources.

\section{References}

Abramson, H., Wen-Hwa, C., and Garza, L. (1963) Liquid sloshing in spherical tanks. AIAA Journal, 1(1), 384-389. 
Bach, P. and Hassager, O. (1985) An algorithm for the use of Lagrangian specification in Newtonian fluid mechanics applications to free surface flows. J. Fluid Mech., 152, 173.

Balendra, T., Ang, K., Paramasivam, P., and Lee, S. (1982) Free vibration analysis of cylindrical liquid storage tanks. Int. J. of Mech. Sciences, 24(1), 47-59.

Bauer, H. (1963) Liquid sloshing in a cylindrical circular tank. AIAA Journal, 1, 2601-2606.

Brebbia, C. (1984) Topics in Boundary Elements Research, vol. 1-5. Springer Verlag.

Chiapada, S., Jue, T., Joo, S., Wheeler, M., and Ramaswamy, R. (1996) Numerical simulation of free-boundary problems. Computational Fluid Dynamics, 7, 91.

Cottle, R. (1974) Manifestions of the Schur complement. Linear Algebra Applications, 8, 189211.

Dutta, S. and Laha, M. (2000) Analysis of the small amplitude sloshing of a liquid in a rigid container of arbitrary shape using a low-order boundary element method. Int. J. for Num. Meth. in Engng., 47, 1633-1648.

Faltinsen, O. (1998) Sea Loads on Ships and Offshore Structures. Cambridge Univ. Press.

Givoli, D. and Keller, J. (1990) A Finite Element Method for Large Domains. Comm. Numer. Meth. Engng., 12, 261-279.

Graham, E. and Rodríguez, A. (1952) The characteristic of fuel motion which affect airplane dynamics. J. of App. Mech., 19, 381-388.

Haroun, M. (1983) Vibration studies and tests of liquid storage tanks. Earthquake Engng. and Structural Dynamics, 11, 179-206.

Housner, G. (1957) Dynamic pressures on accelerated fluid containers. Bulletin of Seismic Society of America, 47, 15-35.

Huan, R. and Thompson, L. (2000) Accurate radiation boundary conditions for the timedependent wave equation on unbounded domains. Int. J. for Num. Meth. in Engng., 47, $1569-1603$.

Huang, Y. and Sclavounos, P. (1998) Nonlinear ship motions. J. of Ship Research, 42(2), 120130.

Hwang, I. and Ting, K. (1987) Dynamics analysis of storage tanks including hydrodynamic interaction by boundary element method. In Transactions of the 9th International Conference on Structural Mechanics in Reactor Technology, 429-434. 
Kawahara, M. and Miwa, T. (1984) Finite element analysis of wave motion. Int. J. for Num. Meth. in Engng., 20, 1193.

Khayat, R., Plaskos, C., and Genouvrier, D. (2001) An adaptive boundary-element approach for 3D transient free surface cavity flow, as applied to polymer processing. Int. J. for Num. Meth. in Engng., 50, 1347-1368.

Koh, H., Kim, J., and Park, J. (1998) Fluid-structure interaction analysis of 3-D rectangular tanks with a variationally coupled BEM-FEM and comparison with test results. Earthquake Engng. and Structural Dynamics, 27, 109-124.

Koshlyakov, N., Smirnov, M., and Gliner, E. (1964) Differential Equations of Mathematical Physics. North-Holland Publishing Co, Amsterdam.

Kuo, C. and Morino, L. (1974) Subsonic potential aerodynamics for complex configurations: a general theory. AIAA Journal, 12, 191-197.

Lloyd, A. (1989) Seakeeping. Ship Behavior in Rough Weather. Ellis Horwood Limited (Chichester).

Maître, T. (1988) Modelisation de l'ecoulémént autour d'une hélice marine par la méthode du potentiel. Ph.D. thesis, Institut National Polytechnique de Grenoble, France.

Moiseev, N. (1964) Introduction to the theory of oscillations of liquid-containing bodies. In Advances in Applied Mechanics, vol. 8, 233-289. Academic Press.

Moiseev, N. and Petrov, A. (1966) The calculation of free oscillations of a liquid in a motionless container. In Advances in Applied Mechanics, vol. 9, 91-155. Academic Press.

Morand, H. and Ohayon, R. (1995) Fluid Structure Interaction. Wiley, Paris.

Morino, L., ed. (1985) Computational Methods in Potential Aerodynamics. Springer-Verlag.

Mueller, A. and Kinnas, S. (1999) Propeller sheet cavitation predictions using a panel method. Journal of Fluids Engineering, 121, 282-288.

Nickell, R., Tanner, R., and Caswell, B. (1974) The solution of viscous incompressible jet and free-surface flows using finite element method. J. Fluid Mech., 65, 189.

Quarteroni, A. (1995) Domain decomposition methods for wave propagation problems. In Keyes, D., Saad, Y., and Truhlar, D., eds., Domain based Parallelism and problem decomposition methods in computational science and engineering. SIAM, Philadelphia.

Ramaswamy, B. and Kawahara, M. (1987) Lagrangian finite element analysis applied to viscous free surface fluid flow. Int. J. Num. Meth. Fluids, 7, 953. 
Ruschak, K. (1980) A method of incorporating free boundaries with surface tension in finite element fluid flow simulation. Int. J. for Num. Meth. in Engng., 15, 639.

Serón, F. and Sabadell, F. (2000) The multiblock method. A new strategy based on domain decomposition for the solution of wave propagation problem. Int. J. for Num. Meth. in Engng., 49, 1353-1376.

Shy, W., Udaykumar, H., Rao, M., and Smith, R. (1996) Computational Fluid Dynamics with Moving Boundaries. Taylor \& Francis.

Siliman, W. and Scriven, L. (1980) Separating flow near a static contact line: slip at the wall and shape of a free surface. J. Comp. Physics, 34, 287.

Williams, B. (1985) The prediction of separated flow using a viscous-inviscid interaction method. Aeronautical Journal, 89(885), 185-197.

York, A., Sulsky, D., and Schreyer (2000) Fluid-membrane interaction based on the material point method. Int. J. for Num. Meth. in Engng., 48, 901-924.

\section{APPENDIX: Annular surface waves}

Choosing cylindrical coordinates $(r, \theta, z)$, the linearized boundary value problem for the velocitypotential function $\Phi=\Phi(r, \theta, z, t)$, is written as

$$
\begin{array}{cc}
\nabla \Phi=0 & \text { in } \Omega ; \\
\partial_{t t} \Phi+g \partial_{z} \Phi=0 & \text { at } z=0 ; \\
\partial_{n} \Phi=0 & \text { at } r=R ; \\
\lim _{z \rightarrow-\infty} \Phi=0 & \text { infinite depth; }
\end{array}
$$

where $g$ is the gravity acceleration, $n$ the normal to the vertical wall of the circular basis, and $\Omega$ is the fluid domain. Performing, as Koshlyakov et al. (1964), a standard variable separation $\Phi(r, \theta, z, t)=\phi(r, \theta, z) e^{i \omega t}$, where $\omega$ is the angular frequency, we have the spatial problem

$$
\begin{array}{cc}
\nabla \phi=0 & \text { in } \Omega ; \\
\partial_{z} \phi-K \phi=0 & \text { at } z=0 ; \\
\partial_{r} \phi=0 & \text { at } r=R ; \\
\lim _{z \rightarrow-\infty} \phi=0 & \text { infinite depth; }
\end{array}
$$

where $K=\omega^{2} / g$ is the wave-number. Employing cylindrical operators in Eqns. $(38, \mathrm{a}-\mathrm{b})$ we have

$$
\begin{array}{cc}
\frac{1}{r} \frac{\partial}{\partial r}\left(r \frac{\partial \phi}{\partial r}\right)+\frac{1}{r^{2}} \frac{\partial^{2} \phi}{\partial \theta^{2}}+\frac{\partial^{2} \phi}{\partial z^{2}}=0 & \text { in } \Omega ; \\
\frac{\partial \phi}{\partial z}-K \phi=0 & \text { at } z=0 ;
\end{array}
$$


but for annular waves $\partial_{\theta \theta} \phi=0$. Then, doing a variable separation $\phi(r, z)=\psi(r) \chi(z)$ in Eqn. $(39, \mathrm{a}-\mathrm{b})$, we have

$$
\begin{array}{cc}
\frac{1}{\psi}\left(\frac{d^{2} \psi}{d r^{2}}+\frac{1}{r} \frac{d \psi}{d r}+\right)+\frac{1}{\chi} \frac{d^{2} \chi}{d z^{2}}=0 & \text { in } \Omega ; \\
\psi\left(\frac{d \chi}{d z}-K \chi\right)=0 & \text { at } z=0 .
\end{array}
$$

Eqn. $(40, a)$ is satisfy when

$$
\begin{gathered}
\frac{d^{2} \psi}{d r^{2}}+\frac{1}{r} \frac{d \psi}{d r}+\gamma^{2} \psi=0 \\
\frac{d^{2} \chi}{d z^{2}}-\gamma^{2} \chi=0
\end{gathered}
$$

where $\gamma$ is the separation constant but, from the free surface boundary condition, Eqn. $(40, b)$, is $\gamma \equiv K$, so

$$
\frac{d^{2} \chi}{d z^{2}}-K^{2} \chi=0
$$

with solutions $\chi=A e^{ \pm K z}$, but due the boundary condition Eqn. $(38, \mathrm{~d})$, we retain

$$
\chi(z)=A e^{K z} \quad ; \quad \text { for } z \leq 0
$$

But eqn. (41,a) is a Bessel one

$$
r^{2} \frac{d^{2} \psi}{d r^{2}}+r \frac{d \psi}{d r}+\left(K^{2} r^{2}-0\right) \psi=0
$$

doing the variable change $x=K r$, we have

$$
x^{2} \frac{d^{2} \psi}{d x^{2}}+x \frac{d \psi}{d x}+\left(x^{2}-0\right) \psi=0
$$

Its solution is $\psi(x)=C_{1} J_{0}(x)+C_{2} Y_{0}(x)$, where $J_{0}, Y_{0}$ are the Bessel functions of zero order, of first and second class, respectively. The only finite solution at the origin is $\psi(r)=A J_{0}(x)$ so, the spatial solution is

$$
\phi(r, \theta, z)=A J_{0}(K r) e^{K z} \quad ; \quad \text { for } z \leq 0
$$

The allowed wave-numbers $K$ are found from the Neumann boundary condition at the vertical wall, Eqn. (38,c), which imposes

$$
\frac{d \psi}{d r}=K \frac{d J_{0}}{d x}=0 \quad ; \quad \text { at } r=R ;
$$

but $d J_{0} / d x=-J_{1}(x)$ and then

$$
J_{1}\left(x_{\alpha}\right)=0 \quad ; \quad \alpha=1,2, \ldots ;
$$

where $x_{\alpha}=\{3.832,7.016,10.173,13.324,16.471 .$.$\} are the zeros of J_{1}(x)$, that is, for radial sloshing modes with null radial velocity at the vertical wall, the radius of the circular cylinder must be located at one of the zeros of the Bessel function $J_{1}(x)$, of first order and first class and, then, the sloshing modes (eigensolutions) are given by Eqn. (36). 